\title{
Phytochemical Profile and in vitro Assessment of the Cytotoxicity of Green and Roasted Coffee Oils (Coffea arabica L.) and their Polar Fractions
}

\author{
Ana Paula Lorenzen Voytena ${ }^{\oplus * 1}$, Regina Celis Lopes Affonso ${ }^{\oplus 1}$, \\ Heloisa da Silva Pitz ${ }^{\oplus 1}$, Fernanda Ramlov ${ }^{\oplus 1}$, Thais Alberti ${ }^{\oplus 1}$, Daniela Sousa \\ Coelho $^{\oplus 1}$, Aline Pereira ${ }^{1}$, Bruno Bachiega Navarro ${ }^{\oplus 1}$, Simone Fanan ${ }^{\circledR 1}$, \\ Marcelo Casagrande ${ }^{2}$, Rosa Maria Ribeiro-do-Valle ${ }^{1}$ and \\ Marcelo Maraschin ${ }^{\oplus}$
}

\author{
${ }^{1}$ Plant Morphogenesis and Biochemistry Laboratory, Federal University of Santa Catarina, \\ Florianopolis, Santa Catarina, Brazil \\ ${ }^{2}$ Regional Cooperative of Coffee Growers in Guaxupé Ltda (Cooxupé), Guaxupé, Minas Gerais, \\ Brazil
}

(Received June 14, 2017; Revised September 12, 2017; Accepted Revised September 12, 2017)

\begin{abstract}
Green Coffea arabica L. seed oil (GCO) has been used as an active cosmetic ingredient in many skin care products, due to its composition and balance of fatty acids. On the other hand, while roasted coffee oil (RCO) is mainly used for imparting aroma in the food industry, there is no data available to suggest its safety in cell-based model systems. In this context, the present study aims to evaluate the chemical composition of GCO, $\mathrm{RCO}$, and their correspondent polar fractions (PFs); and assess their cytotoxicity and antioxidant potential in vitro. RCO and RCO PF exhibited significantly higher amounts of phenolic compounds, when compared to both GCO and GCO PF. In the DPPH assay, after 5 min of incubation, RCO inhibited about $80 \%$ of radicals, while GCO only achieved half of this activity. Similar results were also obtained for their PFs. Upon exposure to GCO, no cytotoxic effects were observed, in fact, there were slight increments in cell proliferation. Nevertheless, cell exposure to RCO led to significant decreases in cell viability. Increases in the concentration of coffee oil PFs were associated with correspondent relevant increased cytotoxicity. Upon hydrogen peroxide-induced oxidative stress, neither GCO nor RCO treatment were effective in protecting cells.
\end{abstract}

Keywords: Coffea arabica L.; coffee oil; cytotoxicity; antioxidant activity. (C) 2018 ACG Publications. All rights reserved.

\section{Plant Source}

Coffee oils were manufactured and provided by the Regional Cooperative of Coffee Growers of Guaxupé Ltda (Cooxupé), Guaxupé, Minas Gerais, southeastern Brazil, and were obtained through a cold-press process of green and roasted coffee beans. All samples were Coffea arabica L.

\footnotetext{
* Corresponding author: E-mail: anavoytena@hotmail.com; Phone: +55 48 3721-4813.
} 
Phytochemical profile and cytotoxicity of green and roasted coffee oils (Coffea arabica L.)

(Rubiaceae) with oil yield of about $7.5 \%$. Roasting conditions were standard medium roasting (11 $\min )$ at $220^{\circ} \mathrm{C}$. Oils were stored at $-20^{\circ} \mathrm{C}$ under modified atmosphere $\left(\mathrm{N}_{2}\right)$ until use.

\section{Previous Studies}

Coffee, traditionally consumed as a beverage, has been the most commercialized food product worldwide for decades. The oils extracted from green (GCO) and roasted (RCO) coffee beans (Coffea arabica L.) are naturally rich sources of valuable bioactive compounds, such as diterpene esters, fatty acids, and unsaponifiable matter, with biological properties that include antioxidant activity [1]. Many cosmetic products containing GCO and/or its isolated compounds have been used to improve skin conditions, especially in anti-aging therapies, helping to regenerate corneum stratum lipids, restructure skin barrier with emollient properties, and protect against damage caused by UVB radiation [2-8]. On the other hand, RCO is mainly used to impart aroma in the food industry, and to the best of our knowledge, no data on RCO assays are available in cell-based model systems. Also, there is a shortage of reports evaluating the safety of coffee oils components/fractions.

\section{Present Study}

In this context, this study aimed to determine the phytochemical profile of GCO, RCO, and their PFs to, in turn, characterize and evaluate coffee biomass for its fatty acids, alkaloids, phenolics, and antioxidant activity. The cytotoxic effects of samples were also analyzed in vitro using murine fibroblasts (BALB/c 3T3, clone a31) as a biological model system and their cell protective effect upon the induction of oxidative stress by $\mathrm{H}_{2} \mathrm{O}_{2}$.

Analyses of GCO and RCO fatty acid composition were carried out through Gas Chromatography - Flame Ionization Detector, or GC-FID. The main fatty acids already described for C. arabica are linoleic (41.2 to $42.6 \%$ ), palmitic (35.2 to $36.7 \%)$, oleic ( 9.5 to $11.9 \%$ ), stearic ( 7.2 to 9.7\%), linolenic (1.3 to $2.7 \%)$, arachidonic (0.3 to $1.5 \%)$, and myristic $(0.2 \%)$ [9]. Previous reports confirm the GCO and RCO fatty acid profiles herein found [6,8,10-11]; however, arachidic, arachidonic and behenic fatty acids were not identified in the analyzed samples. Fatty acids have unique chemical and physical properties that make them suitable for use in cosmetic applications [8]. The use of oils for skin care takes into account their capacity to moisturize the skin, supporting native lipids of the stratum corneum, thus acting as an auxiliary agent to strengthen the barrier function [12]. Coffee oil, mainly composed of triglycerides, essentially made of linoleic, palmitic, and oleic acids, and residual antioxidants, is a source of substances that naturally occur in the skin lipid matrix. Although only GCO is widely disseminated as a cosmetic ingredient, RCO is also an important source of fatty acids and other bioactive compounds and, hence, also a potential target for pharmaceutical and cosmetics industry.

Plant secondary metabolites, such as phenolic compounds, are usually related to adaptive responses to environmental stress conditions; accordingly, they are considered a promising tool in eliminating the causes and effects of skin aging, skin diseases, and skin damage [13]. The total phenolic content was determined with Folin-Ciocalteau reagent in the analyzed samples; GCO and GCO PF presented values in the range between $0.733 \pm 0.09-0.779 \pm 0.04 \mathrm{mg}$ gallic acid equivalents/g oil while RCO and RCO PF presented significantly higher amounts (Tukey's test, $\mathrm{p}<0.0001$ ), between $2.245 \pm 0.12-2.297 \pm 0.05 \mathrm{mg}$ gallic acid equivalents $/ \mathrm{g}$ oil. As a consequence of the polar character of phenolic compounds, it could be speculated that the low concentration of phenolics found in GCO and RCO is related to the lipophilic nature of the oil and the extraction method of these compounds, i.e., cold-press process. Extractive processes with more polar solvents or supercritical $\mathrm{CO}_{2}$ could guarantee a higher yield of phenolics. Studies performed by our research group with coffee beans residual press cake revealed interesting contents of total phenolics in green and roasted aqueous extracts. Coffee beans residual press cake is obtained after the cold press method of extracting the oil fraction. The resultant biomass is claimed to be rich in bioactive compounds of 
interest for human health and cosmetics, with phenolic content ranging from $24.13 \pm 1.45$ to $35.39 \pm$ $3.69 \mathrm{mg}$ gallic acid equivalent/g biomass, to green and roasted residual press cake, respectively [14].

Besides phenolic compounds, coffee is well recognized as a rich source of alkaloids, especially caffeine. The identification of this compound was based on the retention time of the standard through both LC-DAD and mass spectra (HPLC-DAD-ESI-MS/MS). GCO PF and RCO PF presented the same protonated ion $\left([\mathrm{M}-\mathrm{H}]^{+}\right)$at $\mathrm{m} / z 195$ and the same fragmentation pattern by MS/MS, which is characteristic of caffeine (MW $=194 \mathrm{~g} / \mathrm{mol}$ ). Important contents of caffeine were found in both samples, but GCO PF presented higher levels (209.63 $\pm 1.13 \mathrm{mg} / \mathrm{g})$ compared to RCO PF (171.35 $\pm 1.13 \mathrm{mg} / \mathrm{g}$ ). Previous findings report that caffeine contents are highly dependent on the coffee species and are not significantly altered by the roasting process. Taking this into consideration, the lower content of caffeine detected for RCO PF could be related to losses during the sublimation process [15].

Furthermore, the radical scavenging effect of coffee oils and their PFs were examined. The DPPH method has been widely used to determine antiradical/antioxidant activity of phenolic compounds and plant extracts. RCO and its PF presented higher values of radical scavenging, when compared to GCO (Figure 1). Within 5 min of reaction, RCO and its PF achieved about $80 \%$ of DPPH inhibition, while GCO achieved only half of this activity. It is important to note that the discrepancies between GCO PF and RCO PF activities are in accordance with the results obtained through the method herein used to measure total phenolic content. For many years, the Folin-Ciocalteau assay has been used as a measure of total phenolics in natural products, but the basic mechanism is an oxidation/reduction reaction. Therefore, it can be also be considered an evaluation of antioxidant activity [16].
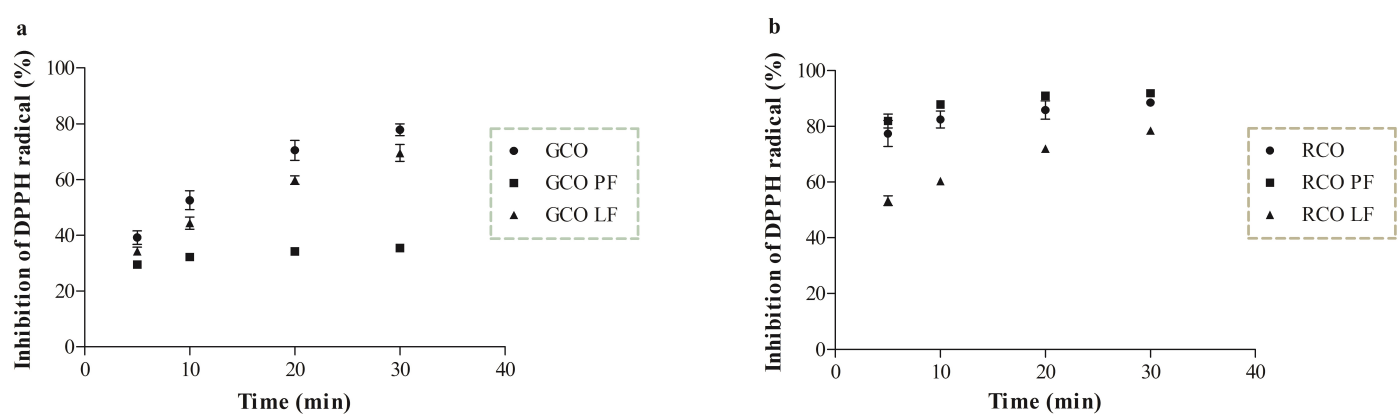

Figure 1 . Radical scavenging activity of $\mathbf{a}$ - green and $\mathbf{b}$ - roasted coffee oils and their lipophilic (LF) and polar (PF) fractions. Values are means \pm standard deviation (SD) of three independent samples performed in triplicate for each one.

The effects of GCO, RCO and their PFs were also analyzed on both cell viability and proliferation using the BALB/c 3T3, clone a31, murine fibroblast cell line. The goal was to detect whether the application of these agents could induce fibroblast proliferation and cytotoxicity towards this cell line. Although botanical extracts have been ingredients commonly added to many cosmetic formulations, rigorous investigation on their efficacy and safety is scarce. Traditionally, the first assay performed to determine safety profile involves in vitro cell viability. In the absence of toxicity, assessment then typically proceeds to in vivo assay.

GCO is becoming increasingly popular in the cosmetics industry, and many products contain it and/or its derived isolated compounds. Moreover, RCO is just as rich as a source of fatty acids, with even higher antioxidant potential. However, no data on RCO assays are available in cell-based model systems. No cytotoxic effects of GCO were detected at the tested doses $(0.001-1 \mathrm{mg} / \mathrm{mL})$, as cell viability was maintained around $100 \%$ in all treatments, with slight increments in cell proliferation (Figure 2). In contrast, after $48 \mathrm{~h}$ treatment with RCO, decreases in cell viability were observed, 
starting at $0.1 \mathrm{mg} / \mathrm{mL}$ and reaching $77 \%$ with $1 \mathrm{mg} / \mathrm{mL}$ at the cell density of $5 \times 10^{3}$ per well. Despite this effect, all other treatments maintained cell viability at around $90 \%$.

a

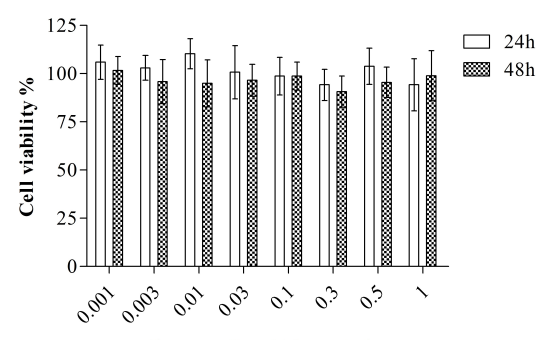

c

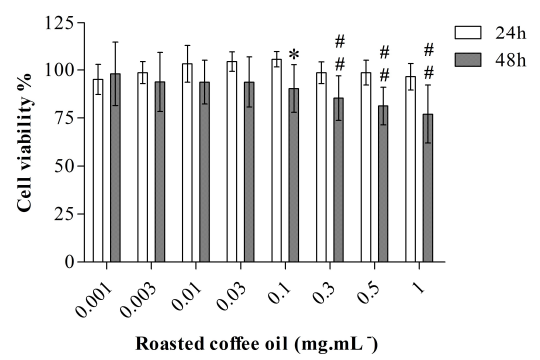

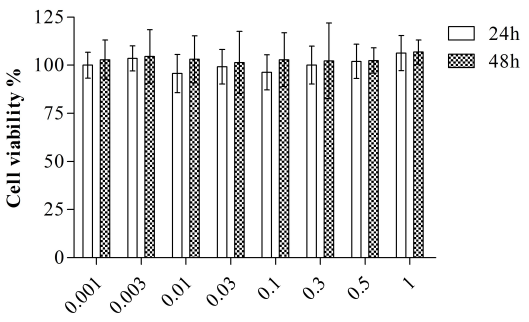

d

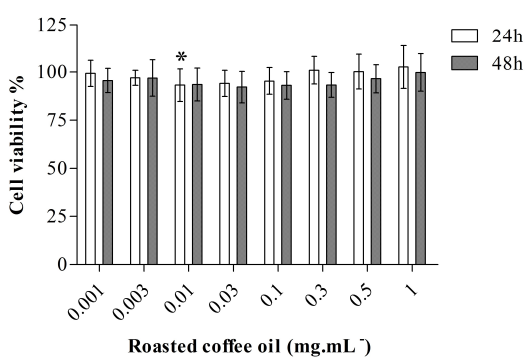

Figure 2. Cell viability measured by the reduction of MTT in BALB/c 3T3, clone a31, fibroblasts after incubation with green and roasted coffee oils $(0.001-1 \mathrm{mg} / \mathrm{mL}) . \mathbf{a}, \mathbf{b}$ - Green coffee oil treatment, cell density $5 \times 10^{3}$ and $1 \times 10^{4}$, respectively; c, $\mathbf{d}$ - Roasted coffee oil treatment, cell density $5 \times 10^{3}$ and $1 \times 10^{4}$, respectively. Data are presented as mean values \pm standard deviation (SD) of three individual experiments performed in sextuplicate. Different letters indicate statistical differences among treatments and control group by ANOVA one-way analyses, followed by Dunnett's post-hoc test. * Indicates $\mathrm{p}<0.05$ for $24 \mathrm{~h}$ treatment and ${ }^{\# \#} \mathrm{p}<0.01$ for $48 \mathrm{~h}$ treatment.

A tendency toward cytotoxicity was detected when cells were treated with coffee oil PFs, starting with $1.25 \mu \mathrm{g} / \mathrm{mL}$ of total phenolic content, reaching low cell viability at $10 \mu \mathrm{g} / \mathrm{mL}$ (Figure 3 ). Based on a literature search, no studies have thus far evaluated the safety of RCO or coffee oil PFs.

Hydrogen peroxide-induced oxidative stress was adopted to determine the antioxidant potential of GCO, RCO, and their PFs on BALB/c 3T3 fibroblast cell line. No tested samples showed effective protection of cells against oxidative stress when added either previously, or concomitantly, to the $\mathrm{H}_{2} \mathrm{O}_{2}$, resulting in a very low rate of cell survival (data not shown), similar to that of control cells. Cytoprotective effects of GCO and RCO PFs were detected when oxidative stress was induced after treatment with the PFs. The absence of protective effects observed for the oils is probably associated with the nonpolar nature of the tested samples and/or the low concentrations that were tested based on the limited solubility of the oils, given that both GCO and RCO showed antioxidant activity.

Our results demonstrated that GCO showed no cytotoxic effects to BALB/c 3T3 fibroblasts. Nevertheless, since cytotoxic effects were shown by coffee oil PFs and RCO, their use in high concentrations should be carefully considered. Our literature search displayed that no cytotoxicity evaluation of RCO and coffee oil PFs is available up to date. Although GCO is claimed to be rich in phenolic content, this is the first study revealing that the oil obtained through a cold-press extraction is in fact low in these metabolites. It implicates that phenolics are possibly trapped within the coffee beans residual press cake, and therefore are not efficiently extracted in the oil with this method. Further studies are required targeting new applications and properties of RCO, but it is important to highlight its higher phenolic content and antioxidant potential. Besides the high content of fatty acids, another bioactive compound of interest derived from coffee oils is the alkaloid caffeine. 

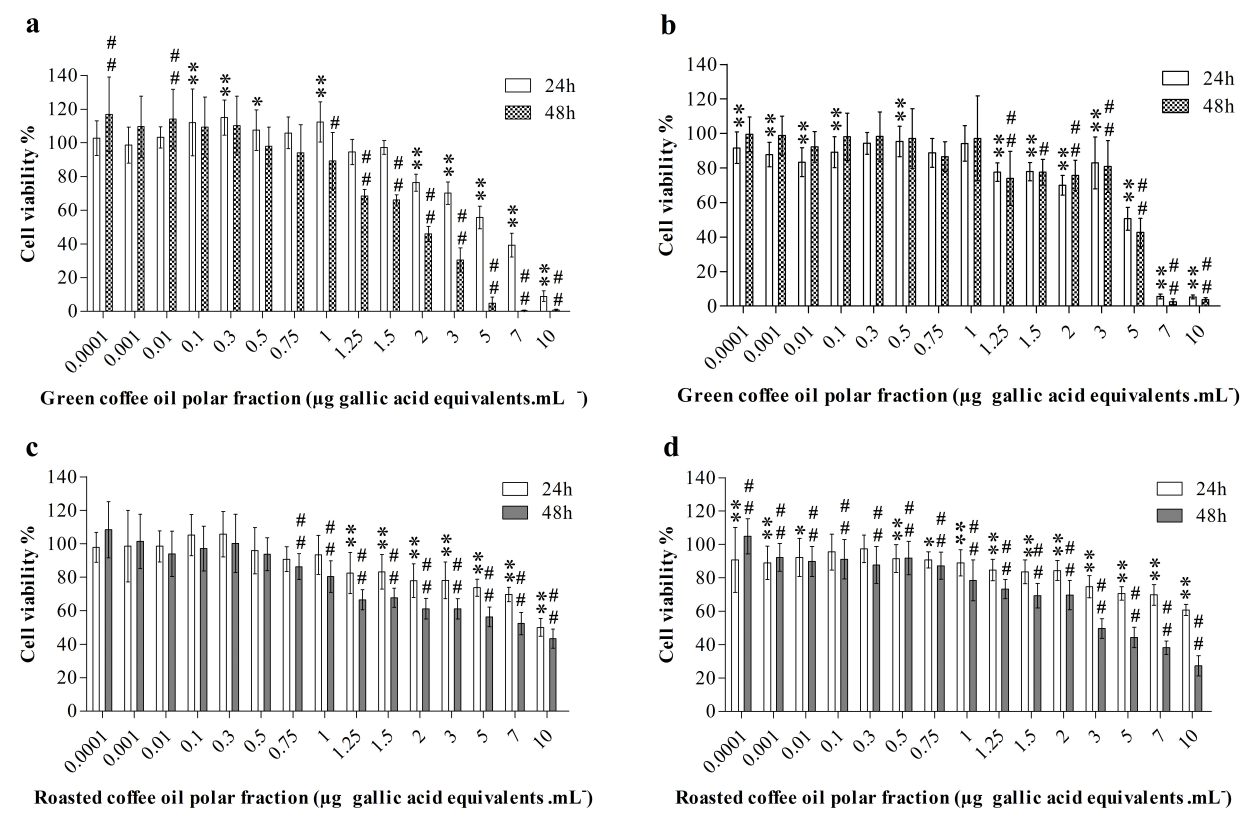

Figure 3. Cell viability measured by the reduction of MTT in BALB/c 3T3, clone a31, fibroblasts after incubation with green and roasted coffee oil polar fractions $(0.0001-10 \mu \mathrm{g}$ gallic acid equivalents $/ \mathrm{mL}$ ). a, b - Green coffee oil polar fraction treatment, cell density $5 \times 10^{3}$ and $1 \times 10^{4}$, respectively; c, d - Roasted coffee oil polar fraction treatment, cell density $5 \times 10^{3}$ and $1 \times 10^{4}$, respectively. Data are presented as mean values \pm standard deviation (SD) of three individual experiments performed in sextuplicate. Different symbols indicate statistical differences among treatments and control group by ANOVA one-way analyses, followed by Dunnett's post-hoc test. * Indicates $\mathrm{p}<0.05$ and $* * \mathrm{p}<0.01$ for $24 \mathrm{~h}$ treatment and ${ }^{\#}$ Indicates $\mathrm{p}<0.05$ and ${ }^{\# \#} \mathrm{p}<0.01$ for $48 \mathrm{~h}$ treatment.

\section{Acknowledgments}

Financial support for this investigation by the National Council for Scientific and Technological Development (CNPq) and Coordination for the Improvement of Higher Education Personnel (CAPES) is acknowledged. The research fellowship from CNPq on behalf of M. Maraschin is also acknowledged. Authors want to acknowledge the Central Laboratory of Structural Molecular Biology (CEBIME) and Multiuser Laboratory of Biology Studies (LAMEB).

\section{Supporting Information}

Supporting Information accompanies this paper on http://www.acgpubs.org/RNP

\section{ORCID}

Ana Paula Lorenzen Voytena: 0000-0002-4411-5526

Regina Celis Lopes Affonso: 0000-0003-1987-2696

Heloisa da Silva Pitz: 0000-0002-8308-7740

Fernanda Ramlov: 0000-0001-7925-3980 
Thais Alberti: 0000-0002-2353-9573

Daniela Sousa Coelho: 0000-0001-5239-1291

Bruno Bachiega Navarro: 0000-0002-2218-5291

Simone Fanan: 0000-0001-7787-7781

Marcelo Maraschin: 0000-0002-4146-4835

\section{References}

[1] H.G. Maier (1981). Kaffee. Paul Parey Verlag, Berlin/Hamburg, Germany.

[2] A.M.R. Alvarez and M.L.G. Rodríguez (2000). Lipids in pharamaceutical and cosmetic preparations, Grasas y aceites 51, 74-96.

[3] M. Athar and S.M. Nasir (2005). Taxonomic perspective of plant species yielding vegetable oils used in cosmetics and skin care products, Afr. J. Biotechnol. 4, 36-44.

[4] M.D.C.V. Pereda, G.C. Dieamant, S. Eberlin, C. Nogueira, D. Colombi, L.C. Di Stasi and M.L.S. Queiroz (2009). Effect of green Coffea arabica L. seed oil on extracellular matrix components and waterchannel expression in in vitro and ex vivo human skin models, J. Cosmet. Dermatol. 8, 56-62.

[5] A.L. Savian, F.T. Varella, M.L. Athayde and C.D.B. Silva (2011). Desenvolvimento e avaliação preliminar da estabilidade de emulsão não-iônica $\mathrm{O} / \mathrm{A}$ contendo óleo de café verde como potencializador de fator de proteção solar, Rev. Bras. Farmácia 91, 82-88.

[6] T.A.L. Wagemaker, C.R.L. Carvalho, N.B. Maia, S.R. Baggio and O. Guerreiro Filho (2011). Sun protection factor, content and composition of lipid fraction of green coffee beans, Ind. Crops Prod. 33, 469-473.

[7] T.A.L. Wagemaker, A.S. Fernandes, P.M. Campos, L.M. Rodrigues and P. Rijo (2012). Evaluation of antioxidant and antimicrobial activities of green coffee oil in cosmetic formulations, Biomed. Biopharm. Res. 9, 207-214.

[8] B.G. Chiari, E. Trovatti, É. Pecoraro, M.A. Corrêa, R.M.B. Cicarelli, S.J.L. Ribeiro and V.L.B. Isaac (2014). Synergistic effect of green coffee oil and synthetic sunscreen for health care application, Ind. Crops Prod. 52, 389-393.

[9] P. Folstar (1985). Lipids, In: Coffee I - Chemistry, ed: R.J. Clarke and R. Macrae, Elsevier Applied Science Publishers, London/New York, pp. 203-220.

[10] H.P. Kaufman and R.S. Hamsagar (1962). Component lipids of the coffee bean. I. Fatty acids esters of cafestol, Fett Wiss Technol. 64, 206-213.

[11] G. Barbiroli (1966). Transformation of fatty substances in coffee during industrial roasting, Quad. Merceol. 5, 223-235.

[12] G.N. Stamatas, J. Sterke, M. Hauser, O. von Stetten, A. van der Pol (2008). Lipid uptake and skin occlusion following topical application of oils on adult and infant skin, J. Dermatol. Sci. 50, 135-142.

[13] M. Dzialo, J. Mierziak, U. Korzun, M. Preisner, J. Szopa and A. Kulma (2016). The potential of plant phenolics in prevention and therapy of skin disorders, Int. J. Mol. Sci. 17, 1-41.

[14] R.C.L. Affonso, A.P.L. Voytena, S. Fanan, H. Pitz, D.S. Coelho, A.L. Horstmann, A. Pereira, V.G. Uarrota, M.C. Hillmann, L.A.C. Varela, R.M. Ribeiro-do-valle and M. Maraschin (2016). Phytochemical composition, antioxidant activity, and the effect of the aqueous extract of Coffee (Coffea arabica L.) Bean Residual Press Cake on the Skin Wound Healing, Oxid. Med. Cell. Longev. 2016, 1-10.

[15] A. Farah (2012). Coffee constituents, In: Coffee: Emerging health effects and disease prevention. ed: Y. F. Chu, John Wiley \& Sons, Oxford, England, pp. 21-58.

[16] R.L. Prior, X. Wu and K. Schaich (2005). Standardized methods for the determination of antioxidant capacity and phenolics in foods and dietary supplements, J. Agric. Food Chem. 53, 4290-4302.

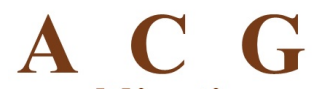

publications

(C) 2018 ACG Publications 